\title{
On the convergence of derivatives of B-splines to derivatives of the Gaussian function
}

\author{
RALPH BRINKS \\ Philips Research Laboratories, Weisshausstrasse 2, 52066 Aachen, Germany \\ E-mail: rbrinks@gmx.de
}

\begin{abstract}
In 1992 Unser and colleagues proved that the sequence of normalized and scaled B-splines $B_{m}$ tends to the Gaussian function as the order $m$ increases, [1]. In this article the result of Unser et al. is extended to the derivatives of the B-splines. As a consequence, a certain sequence of wavelets defined by B-splines, tends to the famous Mexican hat wavelet. Another consequence can be observed in the continuous wavelet transform (CWT) of a function analyzed with different B-spline wavelets.
\end{abstract}

Mathematical subject classification: $43 \mathrm{~A} 15,42 \mathrm{C} 40,65 \mathrm{~T} 60,65 \mathrm{R} 10$.

Key words: B-splines, Gaussian function, Continuous wavelet transform, Mexican hat wavelet, Scalogram.

\section{Introduction}

In 1992 Unser and colleagues proved that the sequence of normalized and scaled B-splines $B_{m}$ tends to the Gaussian function as the order $m$ increases, [1]. In this article the result of Unser et al. is extended to the derivatives of the B-splines. After some definitions and introductions this is done in the second section.

Due to numerical reasons it has become common to use B-splines as analyzing functions (wavelets) in wavelet analysis. Two implications of the convergence result of the second section in wavelet analysis are presented in the third section. First, a certain sequence of wavelets defined by B-splines tends to the 
famous Mexican hat wavelet. Second, similarities in continuous wavelet transform (CWT) can be observed, when a function is analyzed with different B-spline wavelets.

\section{B-splines: definition and some properties}

Let $p \in \mathbb{R}^{\geq 1}$ and $L^{p}(\mathbb{R})$ as usual denote the set

$$
L^{p}(\mathbb{R})=\left\{f: \mathbb{R} \rightarrow \mathbb{C} \mid f \text { measurable, } \int_{-\infty}^{\infty}|f(t)|^{p} \mathrm{~d} t<\infty\right\} .
$$

For $p=2$ and $f, g \in L^{2}(\mathbb{R})$ define the inner product

$$
\langle f, g\rangle:=\int_{-\infty}^{\infty} f(t) \overline{g(t)} \mathrm{d} t
$$

and the norm

$$
\|f\|:=\sqrt{\langle f, f\rangle},
$$

making $L^{2}(\mathbb{R})$ to a Hilbert space. For $f, g \in L^{2}(\mathbb{R})$ the function $f * g$ is defined as

$$
(f * g)(t):=\int_{-\infty}^{\infty} f(t-y) g(y) \mathrm{d} y .
$$

$f * g$ is called convolution product of $f$ and $g$ and is in $L^{2}(\mathbb{R})$.

For $f \in L^{1}(\mathbb{R})$ define the Fourier transform $f^{\wedge}$ and the inverse Fourier transform $f^{\vee}$ as

$$
\begin{aligned}
& f^{\wedge}(\omega):=\int_{-\infty}^{\infty} f(t) e^{-i \omega t} \mathrm{~d} t, \\
& f^{\vee}(\omega):=\frac{1}{2 \pi} \int_{-\infty}^{\infty} f(t) e^{i \omega t} \mathrm{~d} t .
\end{aligned}
$$

This definition can be extended to functions $f \in L^{2}(\mathbb{R})$, see for example [2].

Now let us define the cardinal B-splines:

Definition 2.1. Define

$$
B_{0}(t):= \begin{cases}1, & \text { for } t \in[-1 / 2,1 / 2] \\ 0, & \text { else. }\end{cases}
$$


and let $B_{m}$ be defined as

$$
B_{m}:=B_{0} * B_{m-1}, m \in \mathbb{N} .
$$

Then $B_{m}, m \in \mathbb{N}_{0}$, has the compact support $\left[-\frac{m+1}{2}, \frac{m+1}{2}\right]$ and is in $C^{m-1}(\mathbb{R})$, $\left(C^{-1}(\mathbb{R})\right.$ denotes the set of the piecewise constant functions). The $B_{m}$ are the famous cardinal B-Splines.

B-splines play an important role in computer-aided design (CAD) and signal processing. For an extensive monography see [3].

For all $B_{m}, m \in \mathbb{N}_{0}$, it holds $B_{m} \in L^{1}(\mathbb{R}) \cap L^{2}(\mathbb{R})$ and

$$
B_{m}^{\wedge}(\omega)=\operatorname{sinc}^{(m+1)}(\omega / 2),
$$

where sinc denotes the sinus cardinalis function:

$$
\operatorname{sinc}(t):= \begin{cases}\frac{\sin (t)}{t}, & \text { for } t \neq 0 \\ 1, & \text { for } t=0\end{cases}
$$

After the necessary definitions have been provided, we can come to the main result of this article. Unser and colleagues in [1] have shown that normalized and scaled versions of the B-splines tend to the Gaussian function:

$$
\lim _{m \rightarrow \infty}\left\{\sqrt{\frac{m+1}{12}} B_{m}\left(\sqrt{\frac{m+1}{12}} \cdot x\right)\right\}=\frac{1}{\sqrt{2 \pi}} \exp \left(-\frac{x^{2}}{2}\right),
$$

where the limit may be taken pointwise or in $L^{p}(\mathbb{R}), p \in[2, \infty)$.

Now it is shown that taking the $k$-th derivative of both sides of equation (2.1) is possible. It holds:

Theorem 2.2. Let be $k \in \mathbb{N}_{0}$ then for $m \geq k+1$ the sequence of the $k$-th derivatives $B_{m}^{(k)}$ of the cardinal B-splines converges to the $k$-th derivative of the Gaussian function,

$$
\lim _{m \rightarrow \infty}\left\{\left(\frac{m+1}{12}\right)^{\frac{k+1}{2}} B_{m}^{(k)}\left(\sqrt{\frac{m+1}{12}} \cdot x\right)\right\}=\frac{1}{\sqrt{2 \pi}} \frac{\mathrm{d}^{k} \exp \left(-\frac{x^{2}}{2}\right)}{\mathrm{d} x^{k}},
$$

where the limit may be taken pointwise or in $L^{p}(\mathbb{R}), p \in[2, \infty)$.

The proof of Theorem 2.2 is based on two lemmata. 
Lemma 2.3. Let be $k \in \mathbb{N}_{0}$ and $A: \mathbb{R} \rightarrow \mathbb{R}$ be a two times continuously differentiable function with a positive maximum at $x_{0}$ such that

1. $A\left(x_{0}\right)>0$,

2. $A^{\prime}\left(x_{0}\right)=0$,

3. $A^{\prime \prime}\left(x_{0}\right)=-\alpha^{2} A\left(x_{0}\right)<0, \alpha \neq 0$,

then for the sequence

$$
A_{n}(x)=\left[\frac{1}{A\left(x_{0}\right)} A\left(\frac{x}{\alpha}+x_{0}\right)\right]^{n}=\left[A_{1}(x)\right]^{n}
$$

it holds

$$
\lim _{n \rightarrow \infty}\left\{x^{k} A_{n}\left(\frac{x}{\sqrt{n}}\right)\right\}=x^{k} \exp \left(-\frac{x^{2}}{2}\right),
$$

where the limit is taken pointwise.

Proof. First of all let be $k=0$. Define $L_{n}(x):=\ln A_{n}\left(\frac{x}{\sqrt{n}}\right)$. Then with $t_{n}:=\frac{x}{\sqrt{n}}$ it holds

$$
L_{n}(x)=n \ln \left[A_{1}\left(\frac{x}{\sqrt{n}}\right)\right]=\frac{\ln \left[A_{1}\left(\frac{x}{\sqrt{n}}\right)\right]}{1 / n}=x^{2} \frac{\ln \left[A_{1}\left(t_{n}\right)\right]}{\left(t_{n}\right)^{2}} .
$$

Since it holds $A_{1}(0)=1$ and $A_{1}^{\prime}(0)=0$, for the limit $n \rightarrow \infty$, and hence $t_{n} \rightarrow 0$, we may apply l'Hôpital's rule twice:

$$
\begin{aligned}
\lim _{n \rightarrow \infty} L_{n}(x) & =x^{2} \lim _{n \rightarrow \infty} \frac{A_{1}^{\prime}\left(t_{n}\right)}{2 t_{n} A_{1}\left(t_{n}\right)}=\frac{x^{2}}{2} \lim _{n \rightarrow \infty} \frac{A_{1}^{\prime \prime}\left(t_{n}\right)}{A_{1}\left(t_{n}\right)+t_{n} A_{1}^{\prime}\left(t_{n}\right)} \\
& =\frac{x^{2}}{2} A_{1}^{\prime \prime}(0)=-\frac{x^{2}}{2} .
\end{aligned}
$$

This implies the hypothesis for $k=0$. For $k>0$ the hypothesis follows from the case $k=0$ and the limit theorems (multiplying $x^{k}$ on both sides). 
Lemma 2.4. For $k \in \mathbb{N}_{0}$ and $n \in \mathbb{N}, n>k+2$, there is a constant $c_{k} \in \mathbb{R}^{>0}$ such that for

$$
G_{k}(x):=\chi_{\mathbb{R} \backslash[-1,1]}(x) \frac{c_{k}}{\pi^{2} x^{2}}+\pi^{k}|x|^{k} \exp \left(-x^{2}\right)
$$

it holds

$$
G_{k} \in L^{p}(\mathbb{R}), \forall p \in[1, \infty)
$$

and

$$
\pi^{k}|x|^{k} \cdot\left|\operatorname{sinc}\left(\frac{\pi x}{\sqrt{n}}\right)\right|^{n} \leq G_{k}(x)
$$

Proof. The fact that $G_{k}(x) \in L^{p}(\mathbb{R}), p \geq 1$, may be concluded from $\exp \left(-x^{2}\right)$ $\leq x^{-2 k}$ for $x$ large in modulus. Due to the symmetry we may assume $x \geq 0$. For $x \in[0,1]$ it holds

$$
\sin (\pi x) \leq \pi x-\frac{\pi^{3} x^{3}}{3 !}+\frac{\pi^{5}}{5 !}
$$

and hence $\operatorname{sinc}(\pi x) \leq 1-x^{2}$.

1. Let $x \in[0, \sqrt{n}]$.

Then it holds

$$
\operatorname{sinc}^{n}\left(\frac{\pi x}{\sqrt{n}}\right) \leq\left(1-\frac{x^{2}}{n}\right)^{n} .
$$

Now we show that

$$
\left(1-\frac{x^{2}}{n}\right)^{n} \leq e^{-x^{2}}
$$

Let

$$
p(x):=e^{x^{2}}\left(1-\frac{x^{2}}{n}\right)^{n},
$$

then it holds

$$
p^{\prime}(x)=-\frac{2}{n} x^{3} e^{x^{2}}\left(1-\frac{x^{2}}{n}\right)^{n-1}<0, \forall x \in[0, \sqrt{n}),
$$

and thus $\max _{x \in[0, \sqrt{n}]}=p(0)=1$. 
2. Let $x \in[\sqrt{n}, \infty)$.

Set

$$
c_{k}:=\max _{n>k+2}\left(\frac{n^{(k+2) / 2}}{\pi^{n-k-2}}\right) .
$$

The existence of $c_{k}$ follows from the convergence of the sequence

$$
\left(\frac{n^{(k+2) / 2}}{\pi^{n-k-2}}\right)_{n>k+2}
$$

Then one follows

$$
\begin{aligned}
n^{\frac{k+2}{2}} & \leq c_{k} \pi^{n-k-2} \\
(\sqrt{n})^{k+2}(\sqrt{n})^{n-k-2} & \leq c_{k} \pi^{n-k-2} x^{n-k-2} \\
\left(\frac{\sqrt{n}}{x \pi}\right)^{n} & \leq \frac{c_{k}}{x^{k+2} \pi^{k+2}} .
\end{aligned}
$$

By noting that

$$
\left|\operatorname{sinc}^{n}\left(\frac{\pi x}{\sqrt{n}}\right)\right| \leq\left(\frac{\sqrt{n}}{\pi x}\right)^{n}
$$

the hypothesis follows.

Proof of Theorem 2.2. Set $A(x):=\operatorname{sinc}(x / 2)$, then for $A$ the assumptions of Lemma 2.3 are fulfilled with $\alpha=\frac{1}{\sqrt{12}}$. The lemma states that

$$
x^{k} A_{n}\left(\frac{x}{\sqrt{n}}\right)=x^{k} \operatorname{sinc}^{n}\left(\frac{x}{2} \sqrt{\frac{12}{n}}\right)
$$

tends to $x^{k} \exp \left(-\frac{x^{2}}{2}\right)$ if $n \rightarrow \infty$. Furthermore, it holds $B_{m}^{\wedge}=\operatorname{sinc}^{(m+1)}(\cdot / 2)$, and $B_{m} \in C^{m-1}(\mathbb{R})$. The later yields for $k \leq m-1$ :

$$
\left(B_{m}^{(k)}\right)^{\wedge}(\omega)=i^{k} \omega^{k} \operatorname{sinc}^{(m+1)}\left(\frac{\omega}{2}\right) .
$$


Consequently, one obtains

$$
\begin{gathered}
\left(\frac{m+1}{12}\right)^{\frac{k+1}{2}}\left[B_{m}^{(k)}\left(\sqrt{\frac{m+1}{12}} \cdot\right)\right]^{\wedge}(\omega) \\
=i^{k} \omega^{k} \operatorname{sinc}^{(m+1)}\left(\frac{\omega}{2} \sqrt{\frac{12}{m+1}}\right) \\
\longrightarrow i^{k} \omega^{k} \exp \left(-\frac{\omega^{2}}{2}\right) \text { for } m \rightarrow \infty \\
=i^{k} \omega^{k}\left(\frac{1}{\sqrt{2 \pi}} \exp \left(-\frac{t^{2}}{2}\right)\right)^{\wedge}(\omega) \\
=\left[\frac{\mathrm{d}^{k}}{\mathrm{~d} t^{k}}\left(\frac{1}{\sqrt{2 \pi}} \exp \left(-\frac{t^{2}}{2}\right)\right)\right]^{\wedge}(\omega)
\end{gathered}
$$

where the limit in equation (2.2) follows from Lemma 2.3 and is meant to be taken pointwise. The $L^{p}$-convergence, $p \geq 1$, follows from Lebesgue's Dominated Convergence Theorem, using Lemma 2.4. The hypothesis follows from taking the Fourier transform and the fact that for $p \in[1,2]$ and $1 / p+1 / q=1$ the Fourier transform is a bounded operator from $L^{p}(\mathbb{R})$ to $L^{q}(\mathbb{R})$, see [2, Sect. V.1].

\section{Wavelet analysis}

In this section two implications of Theorem 2.2 for wavelet analysis are presented briefly. For a more detailed treatment of wavelet analysis, see for example the books of Daubechies [4] or Chui [5].

Definition 3.1. A function $\psi \in L^{2}(\mathbb{R}) \backslash\{0\}$ is called a wavelet, iff $\psi$ fulfills the admissibility condition

$$
C_{\psi}:=\int_{-\infty}^{\infty} \frac{\left|\psi^{\wedge}(\omega)\right|^{2}}{|\omega|} \mathrm{d} \omega<\infty
$$

holds. Henceforth, we assume $\psi$ to be normalized: $\|\psi\|=1$. 
Example 3.2. Here we give two examples of famous wavelets:

1. The Haar wavelet is defined by

$$
\psi=\chi_{[0,1 / 2)}-\chi_{[1 / 2,1)}
$$

2. The second derivative of the Gaussian $\exp \left(-\frac{t^{2}}{2}\right)$

$$
\psi(t)=\frac{\mathrm{d}^{2}}{\mathrm{~d} t^{2}} \exp \left(-\frac{t^{2}}{2}\right)=\left(t^{2}-1\right) \exp \left(-\frac{t^{2}}{2}\right)
$$

is another standard wavelet, the Mexican hat wavelet. The normalized version is $\frac{2}{\pi^{1 / 4} \sqrt{3}}\left(t^{2}-1\right) \exp \left(-\frac{t^{2}}{2}\right)$.

\subsection{Convergence to the Mexican hat wavelet}

The construction of the Mexican hat wavelet as a derivative of the Gaussian function may be generalized:

Proposition 3.3. Let $\varphi \in C^{k}(\mathbb{R}) \cap L^{2}(\mathbb{R}) \backslash\{0\}, k \in \mathbb{N}$, with $\varphi^{(m)}(t) \rightarrow 0$ as $|t| \rightarrow 0$ for all $m \in\{0, \ldots, k-1\}$ and $\varphi^{(k)} \in L^{1}(\mathbb{R}) \cap L^{2}(\mathbb{R})$, then

$$
\psi:=\frac{\varphi^{(k)}}{\left\|\varphi^{(k)}\right\|}
$$

is a normalized wavelet.

Proof. First of all $\psi$ cannot be constant zero, which would imply that $\varphi$ is a polynomial, what contradicts the assumptions. Without loss of generality assume $\left\|\varphi^{(k)}\right\|=1$. From Fourier analysis it follows $\psi^{\wedge}(\omega)=(i \omega)^{k} \cdot \varphi^{\wedge}(\omega)$ and hence

$$
\begin{aligned}
\int_{-\infty}^{\infty} \frac{\left|\psi^{\wedge}(\omega)\right|^{2}}{|\omega|} \mathrm{d} \omega= & \int_{|\omega| \leq 1}|\omega|^{2 k-1}\left|\varphi^{\wedge}(\omega)\right|^{2} \mathrm{~d} \omega \\
& +\int_{|\omega|>1} \frac{\left|\psi^{\wedge}(\omega)\right|^{2}}{|\omega|} \mathrm{d} \omega \leq\left\|\varphi^{\wedge}\right\|^{2}+\left\|\psi^{\wedge}\right\|^{2} .
\end{aligned}
$$

The Parseval formula in $L^{2}(\mathbb{R})$ proves that $\psi$ is admissible. 
With a view to numerical calculations wavelets with compact support are mostly preferred. Thus, the derivatives of the cardinal B-splines $B_{m}$, which of course have compact support, are nice candidates for wavelets. For those wavelets, we now can state the first consequence from Theorem 2.2:

Corollary 3.4. For $m \in \mathbb{N}, m \geq 3$, define the normalized wavelets

$$
\psi_{m}:=\frac{B_{m}^{\prime \prime}\left(\sqrt{\frac{m+1}{12}} \cdot\right)}{\left\|B_{m}^{\prime \prime}\left(\sqrt{\frac{m+1}{12}} \cdot\right)\right\|},
$$

then the sequence $\left(\psi_{m}\right)_{m \geq 3}$ converges to the normalized Mexican hat wavelet as $m$ tends to infinity.

\subsection{Similarity in the continuous wavelet transform}

The second consequence of the convergence result of the previous section relates to the continuous wavelet transform (CWT). The CWT is an integral transform, which correlates a given function $f$ to the dilated and translated versions of wavelet $\psi$.

Definition 3.5. Let $f, \psi \in L^{2}(\mathbb{R})$, then define

$$
\begin{aligned}
W_{\psi}[f]: \mathbb{R} \times \mathbb{R}^{\neq 0} \rightarrow \mathbb{R} \\
(\beta, \alpha) \mapsto \frac{1}{\sqrt{|\alpha|}} \int_{-\infty}^{\infty} f(t) \overline{\psi\left(\frac{t-\beta}{\alpha}\right)} \mathrm{d} t .
\end{aligned}
$$

If $\psi$ is a wavelet, $W_{\psi}[f]$ is called continuous wavelet transform (CWT) of $f$.

Remark 3.6. By Cauchy-Schwarz's inequality $W_{\psi}[f]$ is well-defined and bounded.

In the continuous wavelet transform (3.2) the parameter $\alpha \in \mathbb{R}^{\neq 0}$ is the scale or dilation parameter, and $\beta \in \mathbb{R}$ is the translation parameter, which may be interpreted as time. Since $|\psi(t)| \rightarrow 0$ as $|t| \rightarrow 0$, the scale parameter $\alpha$ is proportional to the inverse of the frequency which can be detected by correlating the signal $f$ to $\overline{\psi\left(\frac{t-\beta}{\alpha}\right)}$ : the larger the scale $\alpha$, the wider the window of the wavelet, 
and hence the smaller the corresponding analyzed frequency. The CWT is maximal if the frequency of the input signal $f$ matches that of the corresponding wavelet and the phases coincide.

Hence, for an one-dimensional input signal the transform $W_{\psi}[f]$ is a twodimensional description of the signal with respect to the time $\beta$ and the scale $\alpha$. The $(\beta, \alpha)$-space is called parameter plane and a plot of $\left|W_{\psi}[f](\beta, \alpha)\right|^{2}$ against the parameter plane is called a scalogram. Since we are using real wavelets only, henceforth a scalogram is a plot of $\left|W_{\psi}[f](\beta, \alpha)\right|$ against the parameter plane. Those are often arranged as contour plots where the brightness of each pixel represents the modulus of the CWT, see Figure 1.

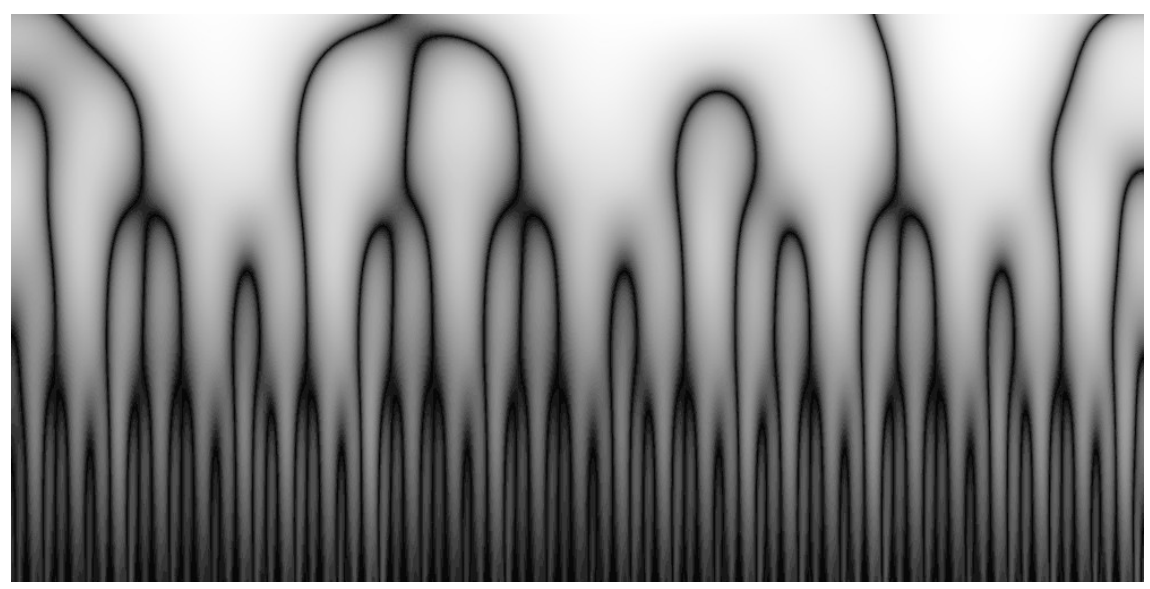

Figure 1 - Scalogram of a function with analyzing wavelet $B_{5}^{\prime \prime}$. The abscissa and ordinate ranges are $\beta \in[0,1]$ and $\log _{2} \alpha \in[-8,-3]$. The gray value of each pixel corresponds to the modulus of the CWT. Black means zero, the brighter the more modulus.

The comparison of the zero-lines of different B-Spline wavelets shows an astonishing similarity for the same order of differentiation. For example the zerolines in the CWT for normalized versions of $B_{4}^{\prime \prime}, B_{5}^{\prime \prime}$ and $B_{6}^{\prime \prime}$ are just vertically shifted as may be seen in Figure 2.

If only the bending points of the zero-lines are plotted in the parameter plane, the shift may be seen more impressively by cascades of three points with the same abscissa values, cf. Figure 3.

The groups of three bending points may be explained by the qualitative similarity of the analyzing wavelets $B_{4}^{\prime \prime}, B_{5}^{\prime \prime}$ and $B_{6}^{\prime \prime}$, see Figure 4 . It is striking that 


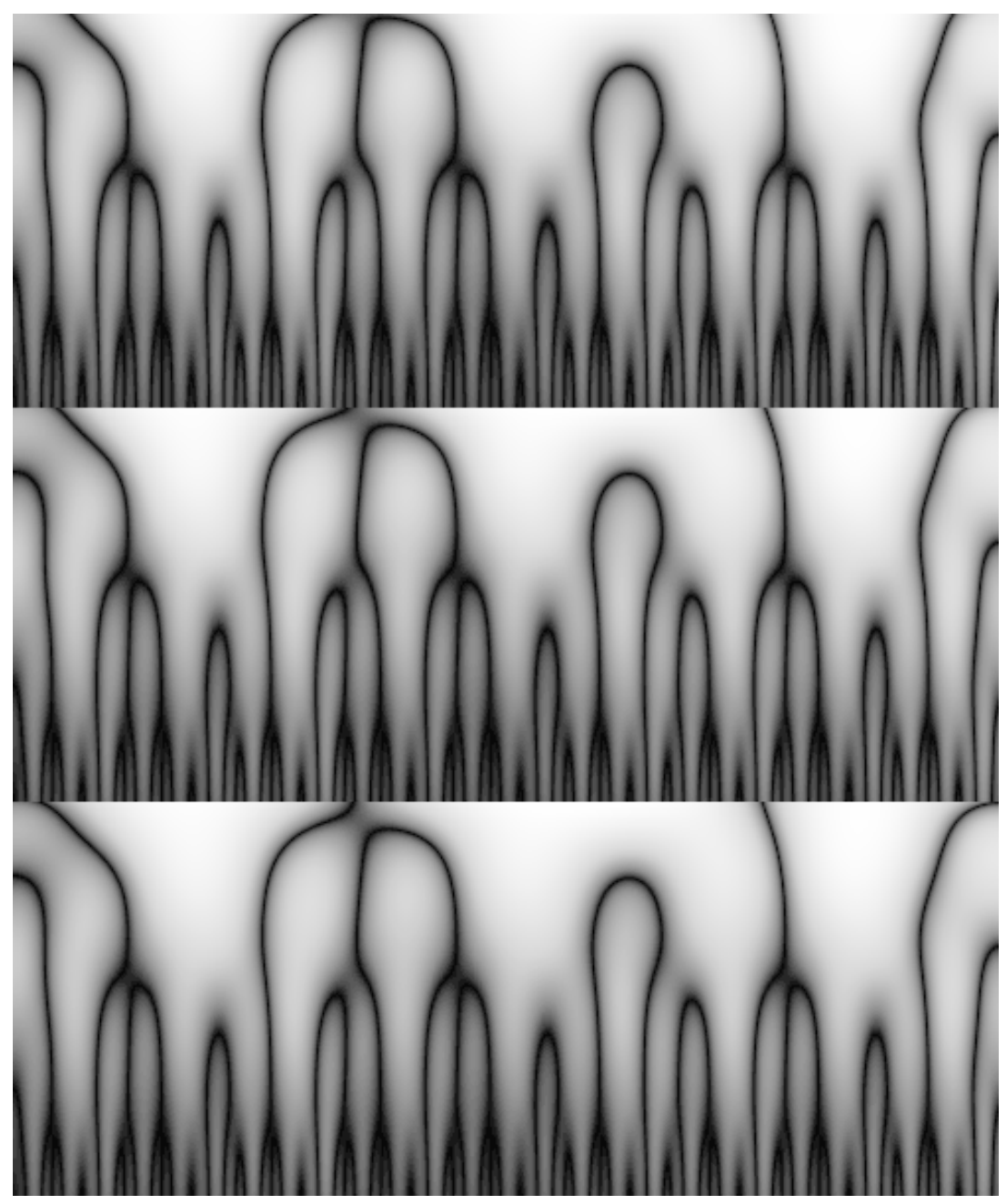

Figure 2 - Scalograms for different analyzing wavelets, from top to down: $B_{4}^{\prime \prime}, B_{5}^{\prime \prime}, B_{6}^{\prime \prime}$, (each normalized). The abscissa and ordinate ranges are $\beta \in[0,1]$ and $\log _{2} \alpha \in$ $[-7,-3]$. Note the vertical shift.

the ratios of the ordinate values of the groups of bending points remain almost constant. Within such a triple group the corresponding ordinates ratios of $B_{5}^{\prime \prime} / B_{4}^{\prime \prime}$ and $B_{6}^{\prime \prime} / B_{5}^{\prime \prime}$ are around 0.92 and 0.93 respectively. To explain this, let $\psi_{1}$ and $\psi_{2}$ be two analyzing wavelets where $\psi_{2}$ is just a scaled (and normalized) version of 


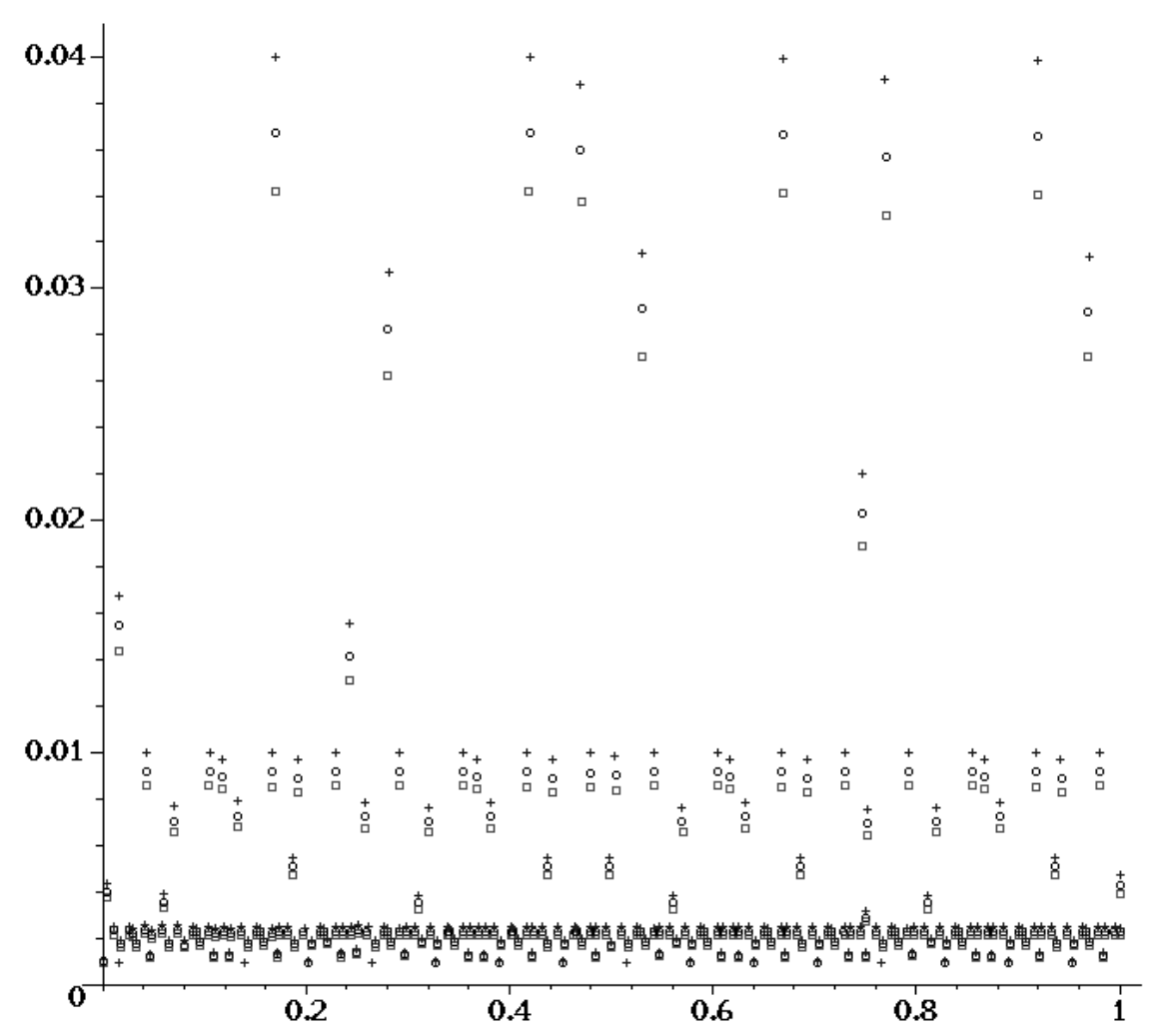

Figure 3 - Bending points for the same data set but different analyzing wavelets $B_{4}^{\prime \prime}(+), B_{5}^{\prime \prime}(\circ)$, and $B_{6}^{\prime \prime}(\square)$.

$\psi_{1}$. Let $s \in \mathbb{R}^{>0}$ and

$$
\psi_{2}(t)=\sqrt{s} \psi_{1}(s \cdot t)
$$

Then it holds

$$
\begin{aligned}
W_{\psi_{2}}[f](\beta, \alpha) & =\frac{1}{\sqrt{|\alpha|}} \int_{-\infty}^{\infty} f(t) \psi_{2}\left(\frac{t-\beta}{\alpha}\right) \mathrm{d} t \\
& =\frac{\sqrt{s}}{\sqrt{|\alpha|}} \int_{-\infty}^{\infty} f(t) \psi_{1}\left(\frac{t-\beta}{\alpha / s}\right) \mathrm{d} t \\
& =W_{\psi_{1}}[f]\left(\beta, \frac{\alpha}{s}\right),
\end{aligned}
$$

Comp. Appl. Math., Vol. 27, N. 1, 2008 
which means that for any $\left(\beta_{0}, \alpha_{0}\right) \in \mathbb{R} \times \mathbb{R}^{\neq 0}$ :

$$
W_{\psi_{2}}[f]\left(\beta_{0}, \alpha_{0}\right)=0 \Leftrightarrow W_{\psi_{1}}[f]\left(\beta_{0}, \frac{\alpha_{0}}{s}\right)=0 .
$$

Consequently, each zero $\left(\beta_{0}, \alpha_{0}\right)$ of $W_{\psi_{2}}[f]$ is shifted to the zero $\left(\beta_{0}, \frac{\alpha_{0}}{s}\right)$ of $W_{\psi_{1}}[f]$.

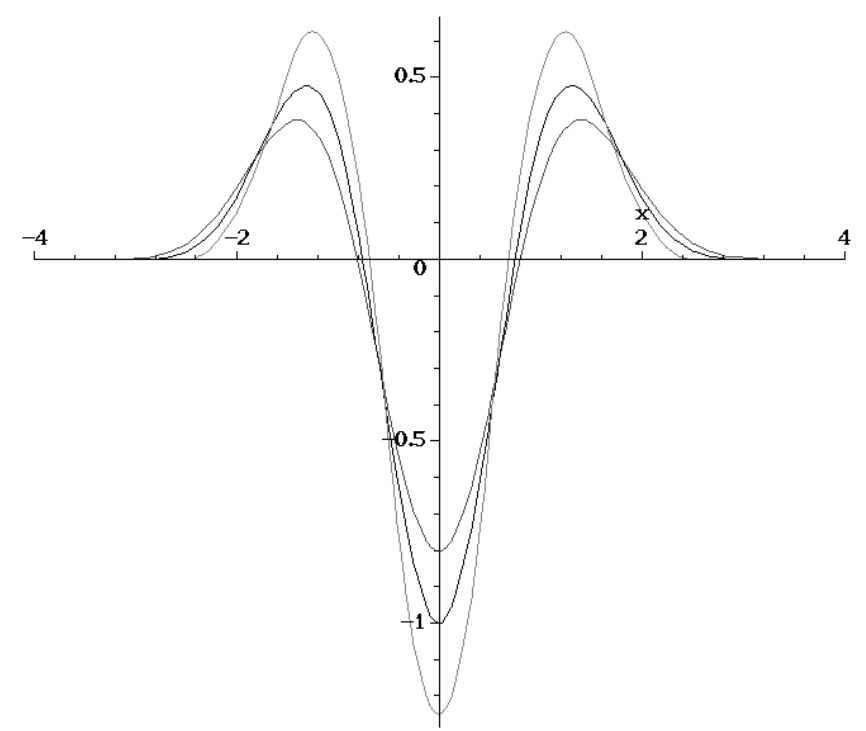

Figure $4-$ Qualitative similarity of $B_{4}^{\prime \prime}, B_{5}^{\prime \prime}$ and $B_{6}^{\prime \prime}$.

Theorem 2.2 explains the vertical shifts of the scalograms by values around 0.9 . For $m=4$ and $m=5$ respectively the ratios of the coefficients inside the arguments $\sqrt{\frac{m+1}{12}}$ of $B_{m}^{\prime \prime}$ and $B_{m+1}^{\prime \prime}$ are $\sqrt{5 / 6} \approx 0.913$ and $\sqrt{6 / 7} \approx 0.926$ respectively.

\section{Summary}

In this article it has been shown that a convergence result of the sequence of B-splines of increasing order to the Gaussian function can be generalized to the derivatives. Since derivatives of B-splines are often used as wavelets in the continuous wavelet transform (CWT), this generalization has implications in the field of wavelet analysis. First, the sequence of the second order derivatives 
of the B-splines tends to the Mexican hat wavelet, and second, the CWTs of a function with different B-spline wavelets have astonishing similarities.

\section{REFERENCES}

[1] M. Unser, A. Aldroubi and M. Eden, On the Asymptotic Convergence of B-Spline Wavelets to Gabor Functions. IEEE Trans. Inform. Theo., 38(2) (1992).

[2] E.M. Stein and G. Weiss, Introduction to Fourier Analysis on Euclidean Spaces. Princeton University Press, Princeton, 1975.

[3] C. de Boor, A Practical Guide to Splines. Springer-Verlag, New York, 1978.

[4] I. Daubechies, Ten Lectures on Wavelets. SIAM, Philadelphia, 1992.

[5] C.K. Chui, An Introduction to Wavelets. Academic Press, Boston, San Diego, 1992. 